\section{TORSION OF THE GALL BLADDER.} BY

L. R. FIFIELD, F.R.C.S.ENG., SURGICAL FIRST ASSISTANT AND REGISTRAR, LONDON HOSPITAL.

Tonsion of the gall bladder is a very rare condition, and in consequence I report the following case.

A married woman, aged 54, with six children, whilst taking tea on February 17th, was seized with severe pain in the right upper abdomen. She vomited frequently bright green bile, and the pain and vomiting continued until the morning of February 19th, when she was admitted to the London Hospital. She had not previously had similar pain, nor flatulent dyspepsia, jaundice, or any abdominal condition, although, until seven years ago, she was alleged to have suffered from periodic "bilious attacks," consisting of headache, slight sickness, but no pain, lasting for about a day. She was always constipated.

Tay. She was always constipated.
The temperature on admission was $99^{\circ}$, pulse 94 , and respirations 24 . The abdomen was slightly distended, and did not move well on respiration. Tenderness and rigidity of the right upper abdomen were pronounced, and a rounded lump, suggestive of a gall stone in Hartmann's pouch obstructing the cystic duct was the clinical diagnosis.

Operation.-A right upper rectus incision was made and the rectus muscle retracted outwards. As soon as the peritoneal cavity was opened some serous fluid escaped and a black distended gall bladder presented. It was about $3 \frac{1}{2}$ in. long, the shape of a thick sausage, with the concavity directed upwards and forwards. The peritoneum completely covered it and attached its proximal end to the liver by a mesentery, which also contained the cystic duct. A cystico-duodenal fold ran from the under surface of the proximal end (the cystic duct end) to the duodenum. The gall proximal had rotated once completely on the cystic duct from right to left. The gall bladder was extremely mobile, and its wall in the region of Hartmann's pouch so thickened as to suggest to the palpating fingers a stone therein. I removed the gall bladder and explored the biliary passages, but found nothing else abnormal.

The gall bladder contained blood mixed with a little bile but no stones. The wall was greatly thickened by haemorrhage, especially at the proximal end, where it measured a quarter to a third of an inch. The patient made an uninterrupted recovery. I am indebted to Sir Hugh Rigby for permission to publish this case.

Relation of the Peritoneum to the Gall Bladder.

The gall bladder lies in a groove on the under surface of the liver between the right and quadrate lobes. Generally the postero-inferior surface and fundus are covered by peritoneum, and the antero-superior surface is attached to the liver by fibrous tissues. Sometimes the gall bladder is crossed by a bridge of liver tissue, and may even be entirely embedded. Rarely it is completely surrounded by peritoneum and attached to the liver by a mesentery. Lett quotes Brewer as finding this condition in 5 out of 100 dissections of the gall bladder and ducts, while $\mathbf{M r}$. A. J. Walton observed it in 7 of his cases at operation. Very rarely this mesentery does not extend beyond the proximal end (" neck") of the gall bladder, so that nearly the whole of the latter is entirely unconnected with the liver. In such cases the gall bladder is extraordinarily mobile and liable to torsion. It is suggested by $\mathbf{M r}$. Walton that a mesentery is more common in cases of virginal ptosis. Not infrequently, especially in cases of ptosis, a peritoneal fold passes from the inferior surface of the gall bladder to the colon or duodenum or both. It is known as the cysticocolic or duodenal fold, and is a continuation of the gastrohepatic omentum. It was present in three of Brewer's five cases of gall bladders with mesenteries, and also in the case of torsion here recorded.

\section{Previous Cases of Torsion.}

Wendel in 1898 described the case of a woman, aged 23, who had torsion of the gall bladder, in which there were stones, whilst other stones had escaped through a perforation and were lying free in the surrounding abscess cavity. The cystic duct had twisted many times, but there was no gangrene of the gall bladder, presumably on account of the many adhesions present.

In 1909 Lett recorded a case in which the patient, aged 72, was found to have her cystic duct twisted four half:turns, but there were no stones present. She died twelve hours after operation.

In the M cdical Annual of 1913, in the section on volvulus of the all bladder, Moynihan and Upcott quote Kubig, who in 1912 published his own case, found post mortem, and the records of three others found in the literature-namely, those of Mühsam, Mayer, and Fischer. These four cases occurred in elderly women. The gall bladder had rotated once or twice completely from right to left, was kidney-shaped, full of blood, and its wall was greatly thickened. In one of the four gall stones were present.
Hansen's case in 1921 was that of a woman, aged 79, who had an hour-glass gall bladder; whilst in 1921 Irwin published the case of a woman, aged 34 , who on operation was found to have a volvulus, there being one complete twist in the proximal part of the gall bladder itself and not the cystic duct. No stones were present and she recovered.

Jonas operated on a woman, aged 67 , in 1923, and found two half-twists of the cystic duct and stones in the gall bladder. She recovered.

Summary.

In all the recorded cases the patients were women and the majority of advanced age. In just over a quarter (3 out of 11) stones were present.

The physical signs and symptoms were practically the same in nearly every case: sudden onset of severe pain in the right upper abdomen, vomiting, slight abdominal distension, rigidity most marked in the right hypochondrium, and a tender lump in the position of the gall bladder. In no instance was a correct diagnosis made, the condition generally being considered acute cholecystitis with a stone obstructing the cystic duct.

Although in nearly all cases the gall bladder was strangulated and gangrene inevitable, apparently (for example, Wendel's case) it may obtain a sufficient blood supply through adhesions to prevent this.

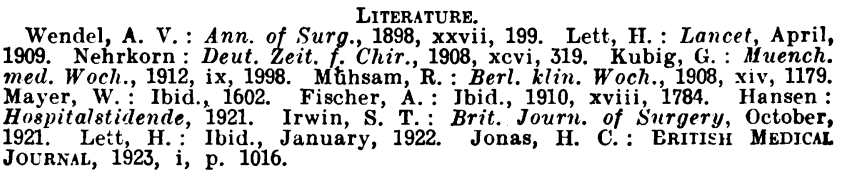
1921. Lett, H.: Ibid.,

\section{OTITIC INTRACRANIAL INFECTION.} BY

H. LAWSON WHALE, M.D., F.R.C.S.,

SURGEON (EAR, NOSE, AND THROAT), LONDON TEMPERANCE HOSPITAL AND HAMPSTEAD GENERAL HOSPITAL.

As a sequel to otorrhoea, with or without mastoid involvement, the presence of pus in the cerebro-spinal fluid is a condition from which recovery is unfortunately not so common as to be uninteresting; and when the surgeon is not only faced with obvious meningitis, but also, in obedience to strong indications of brain abscess, is obliged to explore cerebrum and cerebellum, the patient's prospects are certainly not improved.

Recently at a hospital of 120 beds (the London Temperance) I had simultaneously under my care two cases of this type; and each made a quite unexpected recovery. The following are brief reports.

\section{CASE I.}

A girl, aged 17, had had a discharge from the right ear for two years. In August, 1924, a radical mastoid operation (right) was performed. A month later she complained of giddiness and intense frontal headache; right external strabismus was present, with spontaneous nystagmus of the first degree on deviation to the left; there was indefinite thickening over the right jugular vein, and oedema of both optic discs. At first rigors were present; later and oedema of both optic and respirations fell to subnormal. Pus was found in the lumbar cerebro-spinal fluid.

Operation.- The dura mater was widely exposed, and found to be under slight tension; the wall of the lateral sinus was rough and unhealthy. The sinus was explored with a needle, but only normal blood was extracted. At a second operation, two days later, the dura was very tense and quite devoid of pulsation; first the cerebrum, and then the cerebellum, was explored for pus-without

Post-operative History.-Spasticity of the left psoas, adductors, and hamstrings supervened and persisted for a month, then gradually cleared. Hernia cerebri slowly subsided. The intense
headache was controlled by heroic doses of morphine and heroin. The patient was discharged well.

\section{CASE II.}

A girl, aged 3, in November, 1924, underwent myringotomy for A girl, In January, 1925, she was admitted to right acute otitis media. In January, 1925, she was admitted to hospital suffering from right mastoiditis, and a cortical mastoid
operation was performed. Two days later she was isolated for operation was performed. Two days later she was isolated for
pertussis. Four days later still the head was retracted, and there. were convulsions of the right limbs, with paresis of the right side of the face and complete paralysis of the left arm. Pus was found in the lumbar cerebro-spinal fluid.

Operation.-Chloroform was the only anaesthetic employed, ether being contraindicated on account of cough. The dura mater was widely exposed, and found under slight tension. The wall of the sigmoid sinus was healthy. At a second operation, two days later, the dura was very tense, there was no pulsation whatever. Both 